A R TIICULLO D E I $N$ VES T I G A C I Ó $N$ $\begin{array}{lllllllllllllllllll}C & I & E & N & T & I & F & I & C & A & Y\end{array}$

T E C N O L Ó G I C A

\section{REPRESENTACIONES SOCIALES SOBRE UNIVERSIDAD PÚBLICA DE ESTUDIANTES del Centro tutorial San JuAn NEPOMUCENO ${ }^{1}$ DE LA UNIVERSIDAD de Cartagena}

\author{
SOCIAL REPRESENTATIONS ON PUBLIC UNIVERSITY OF STUDENTS \\ OF THE UNIVERSITY OF CARTAGENA SAN JUAN NEPOMUCENO \\ TUTORIAL CENTER
}

Por: Zoahamy Ardila Ramos*

*Trabajadora Social de la Universidad de Cartagena, especialista en Gerencia del Talento Gerencia del Talento versidad Tecnológica de Bolívar, optando a titulo de Magíster en Educación, Universidad de Cartagena (SUECARIBE): docente y jefa del departamento academico de la Facultad de Ciencias Sociales Educación de la Universidad de Cartagena; miembro del grupo de investigación Educación, Universidad y Sociedad, de la Universidad de Cartagena. mail: zardilar@unicartagena.edu.co

1 Municipio colombiano ubicado en el centro del Departamento de Bolívar, limita al norte con los municipios de El Guamo y Calamar, al este con el municipio de Mahates, al oeste los municipios de Zambrano y San Jacinto, a sur con los municipios de San Jacinto y María la Baja.

\section{RESUMEN}

El presente artículo pretende dar cuenta de los avances del estudio referido a las representaciones sociales sobre universidad pública de estudiantes del centro tutorial San Juan Nepomuceno; de la Universidad de Cartagena (Colombia), las cuales fueron identificadas a través del análisis discursivo, expresado en entrevistas semiestructuradas dirigidas a estos en la etapa de recolección de información primaria; los principales hallazgos dan cuenta de una construcción lingüística acrítica de la categoría: Universidad Pública, evidenciándose representaciones sociales descontextualizadas del enfoque de derecho.

Palabras clave: representaciones sociales, universidad pública, educación superior.

\section{ABSTRACT}

This article intends to give an account of the advances of the study related to social representations on the public university of students of the University of Cartagena tutorial center in San Juan Nepomuceno (Colombia); Which were identified through discursive analysis, expressed in semistructured interviews addressed to them in the stage of primary information collection; The main findings show an uncritical linguistic construction of the category: Public University, demonstrating social representations decontextualized from the right approach.

Keywords: Social representations, public university, higher education.

\section{Introducción}

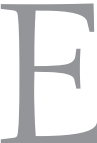

n la actualidad América Latina enfrenta el desafío de brindar sistemas educativos que garanticen el desarrollo, y la sostenibilidad de sus naciones. De cara a este reto, Colombia se asume como un Estado Social de Derecho, organizado en forma de República unitaria, descentralizada, 


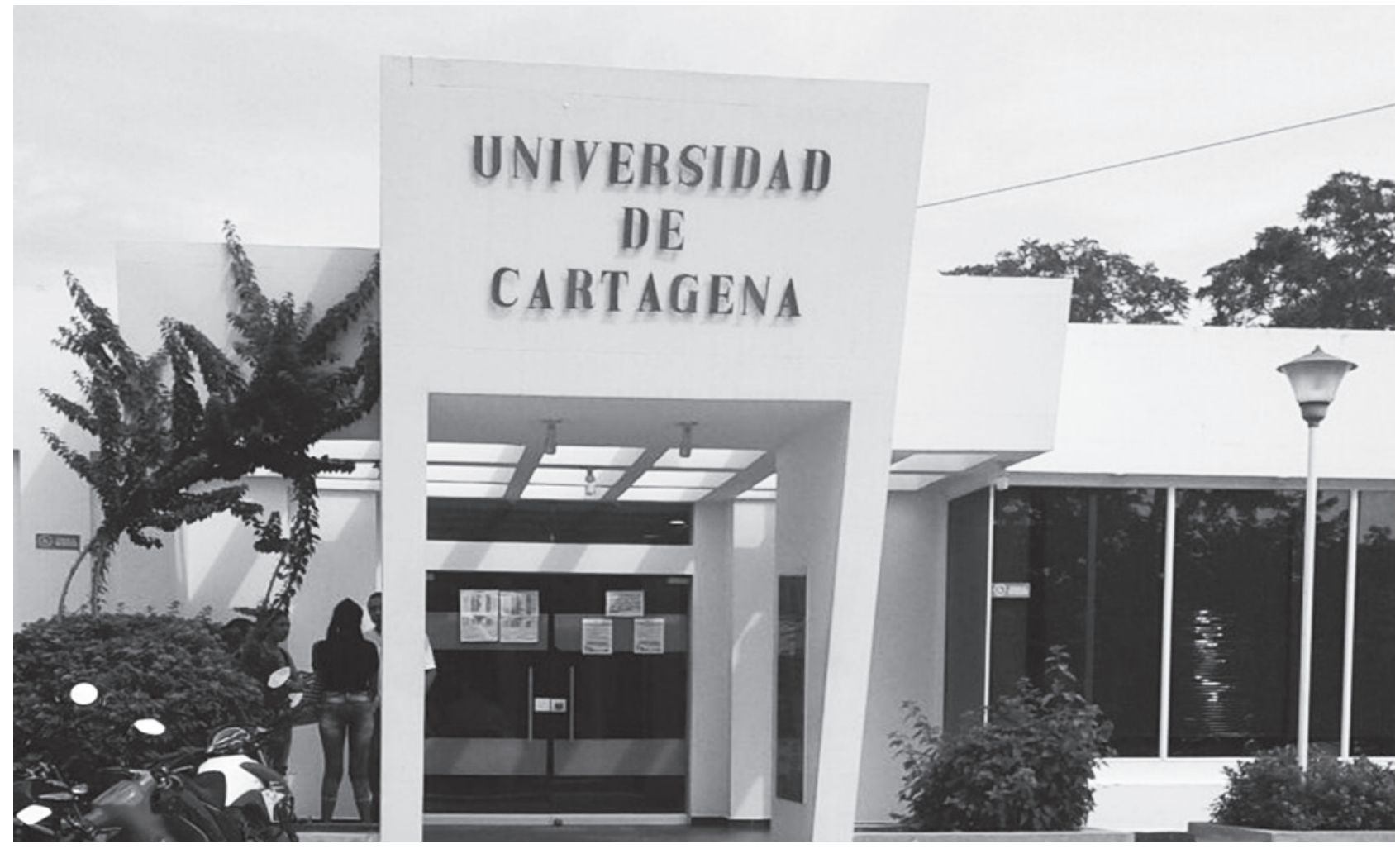

Centro Tutorial San Juan Nepomuceno - Bolívar. 2016 Foto: Zoahamy Ardila Ramos

autónoma, democrática, participativa y pluralista, fundada en el respeto de la dignidad humana y en la prevalencia del interés general ${ }^{2}$. Siendo fines esenciales del Estado colombiano: servir a la comunidad, promover la prosperidad general y garantizar la efectividad de los principios, derechos y deberes consagrados en la Constitución.

De acuerdo a lo anterior, en Colombia se reconoce que la educación es un derecho de la persona y un servicio público que tiene una función social; con ella se busca el acceso al conocimiento, la ciencia, la técnica, y a los demás bienes y valores de la cultura. La educación formará al colombiano en el respeto a los derechos humanos, a la paz y a la democracia; y en la práctica del trabajo y la recreación, para el mejoramiento cultural, científico, tecnológico y para la protección del ambiente. El Estado, la sociedad y la familia son responsables de la educación, que será obligatoria entre los cinco y los quince años de edad.

Asumida la educación como un elemento esencial para la transformación 2 Constitución Política de Colombia Título I. De los principios funsocial, y orientados los procesos educativos hacia la integralidad, la ampliación 
3 Ley General de Educación (Ley 115 de 1994). Ministerio de Educación Nacional.

4 Colombia. Corte Constitucional. Relatoría Sentencia T-068/12

Derecho fundamental a

la educación-Reitera-

ción de jurispruden-

cia. Recuperado de:

http://www.corte-

constitucional gov.co

RELATORIA/2012/T-O 68-12.htm. de cobertura y la calidad, el Ministerio de Educación Nacional mediante la Ley General de Educación ${ }^{3}$, define la gratuidad de la educación en las instituciones del Estado; siendo deber de este, promover y fomentar el acceso a la cultura de todos los colombianos en igualdad de oportunidades, por medio de la formación permanente y la enseñanza científica, técnica, artística y profesional en todas las etapas del proceso de creación de la identidad nacional.

Si la educación es un derecho fundamental que goza de un carácter progresivo ${ }^{4}$, su fundamentalidad está dada por su estrecha relación con la dignidad humana, en su connotación de autonomía individual, ya que su práctica conlleva a la elección de un proyecto de vida y la materialización de otros principios y valores propios del ser humano; y su progresividad la determina: la obligación del Estado de adoptar medidas, en un plazo razonable, para garantizar el ejercicio del derecho, de manera que la simple actitud pasiva de este se opone al principio en mención (aquí encontramos la obligación del Estado de procurar el acceso progresivo de las personas a las universidades, mediante la adopción de ciertas estrategias, dentro de las cuales se encuentran facilitar mecanismos financieros que hagan posible el acceso de las personas a la educación superior, así como la garantía de que progresivamente el nivel de cupos disponibles para el acceso al servicio se vayan ampliando); la obligación de no imponer barreras injustificadas sobre determinados grupos vulnerables y la prohibición de adoptar medidas regresivas para la eficacia del derecho concernido.

Es por ello que, en el Plan Sectorial de Educación 2006-2010: Revolución Educativa, se plantea: El énfasis en la educación como un vehículo para lograr una sociedad más equitativa se tradujo en resultados en términos de ampliación de cobertura y mejoramiento de la calidad. Sin embargo, el país requiere, además de más y mejor educación, que ésta sea más pertinente frente a las demandas de los sectores productivos en una economía globalizada. Motivo por el cual, el énfasis se hace en la educación como una herramienta para construir un país más competitivo, que permita brindar una mejor calidad de vida a sus habitantes.

Ahora bien, en Colombia, la educación superior no está exenta de lo dicho en párrafos anteriores, es regulada por la ley 30 de 1992 en la que queda definida como: "un servicio público cultural, inherente a la finalidad social del Estado". En ese sentido, la universidad pública constituye un patrimonio cultural de las sociedades, cuyo principal activo se concentra en la capacidad 
de servir como medio de emancipación de los universitarios y ciudadanos; y su misión, que es el conocimiento debe garantizar su democratización y universalización.

En concordancia con lo ya planteado, la Educación Superior constituye "un proceso permanente que posibilita el desarrollo de las potencialidades del ser humano de una manera integral... con posterioridad a la educación media o secundaria" ${ }^{5,}$ convirtiendose en un motor de movilidad social y de transformacion para la vida de los estudiantes en los diferentes contextos socioculturales.

Sin embargo, solo hasta hace diez años, el acceso a la Educación Superior era mucho más limitada de lo que es hoy, puesto que la oferta de universidades en las grandes capitales, como Bogotá, Medellín, Cali, Barranquilla, Cartagena, entre otras, no cubría extensas zonas del territorio en donde la demanda era importante. La única opción para estudiar una carrera era, entonces, emigrar a las grandes ciudades.

Atendiendo a lo anterior, y con la finalidad de cerrar las brechas en acceso y calidad al sistema educativo entre los individuos, los grupos poblacionales y entre las regiones, permitiendo a su vez un acercamiento del país a altos estándares internacionales y logrando la igualdad de oportunidades para todos los ciudadanos a lo largo y ancho del territorio, como una de las funciones sustantivas de la educación universitaria en Colombia, y bajo la necesidad de garantizar el pleno goce de la educación superior como un derecho a la población rural. El Ministerio de Educación Nacional de Colombia (Decreto 1295 de 2010, en el Artículo 16) implementa la educación a distancia como una modalidad pedagógica que surge en el contexto social como una solución a los problemas de cobertura y calidad que aquejan a un número elevado de personas, quienes deseaban beneficiarse de los avances pedagógicos, científicos y técnicos que habían alcanzado ciertas instituciones, pero que eran inaccesibles por la ubicación geográfica o bien por los elevados costos que implicaba un desplazamiento frecuente o definitivo a esas sedes.

En consonancia, en el año 1990 con la aprobación de la estampilla pro cultura, que entre sus objetivos contempla la extensión de la universidad pública a otras regiones, la Universidad de Cartagena implementa mediante expedición del Acuerdo 20 del mismo año, emanado por el Consejo Superior, el

5 Ministerio de Educación Nacional República de Colombia. Ley $30 \mathrm{de}$ 1994. Título Primero. Capítulo I. Artículo1 ${ }^{\circ}$ 
6 Piñeres de la Ossa,

Dora. La Cátedra

Historia de Cartagena,

mirar su historia para

construir su futuro.

Volumen 1. Cartagena

de Indias: Editorial

Antillas.
Sistema de Educación Abierta y a Distancia (SEAD), atendiendo a un proyecto nacional para desarrollo de la Educación Superior del Banco Interamericano de Desarrollo -BID- para la costa Caribe; se inauguran los programas de Administración en Salud y Tecnología de Alimentos y se da inicio al proceso de descentralización de los programas de la Universidad de Cartagena hacia el sur del departamento de Bolívar..." ${ }^{6}$

Sin embargo, solo hasta el año 2008 se da la apertura del Centro Tutorial en el municipio de San Juan Nepomuceno, en concordancia con las políticas de regionalización y expansión de la Universidad, a través de la firma de convenio interinstitucional ( 7 de marzo de 2008) suscrito entre el municipio y la Universidad de Cartagena, para la apertura de los programas de pregrado y de educación continuada. Pese a los esfuerzos institucionales por garantizar cobertura y accebilidad, los indices de deserción en este centro son elevados; en contraste con lo expresado el Ministerio de Educación Nacional (2009) reconoció que "El abandono universitario en los programas de distancia, obedece al desconocimiento de los estudiantes de las políticas y estrategias de fomento a la permanencia estudiantil". Para el caso particular del Centro Tutorial de San Juan Nepomuceno, solo el 7\% de los bachilleres del municipio tiene la posibilidad de ingreso al sitema de educación superior, y de cada 10 estudiantes solo 8 culminan de manera satisfactoria la carrera universitaria, según datos suministrados por la Oficina de Planeación Municipal.

Frente al panorama anterior, y a pesar de las institucionalizadas políticas de retención y permanencia, la no existencia de una Oficina de Bienestar Universitario en el Centro Tutorial de San Juan Nepomuceno, la poca o nula socialización de la política pública en materia de educación superior, la débil incorporación del concepto de ciudadano y ciudadanía en los estudiantes de este municipio, se ha constituido en una gran limitante para el conocimiento y exigencia de la comunidad académica, de sus derechos y del goce de las disponibilidades (programas de apoyo académico, psicosocial y socioeconómico) que ofrece la universidad pública a sus estudiantes, elementos que hacen parte de la configuración y construcción de las representaciones sociales que los estudiantes actores de esta investigación asumen de esta categoría.

La presencia de la universidad pública en la región, las concepciones e ideas que tienen los estudiantes de este municipio con respecto a esta, su impacto 
institucional y la interacción de la Universidad con la sociedad desde su compromiso con el desarrollo regional, llevan a preguntarse icuáles son las representaciones sociales que han construido los estudiantes de pregrado de la Universidad de Cartagena, modalidad a distancia del Centro Tutorial San Juan Nepomuceno con respecto a la universidad pública y su incidencia en la deserción estudiantil?

En este artículo se presentan algunos aspectos relevantes de la investigación, llevada a cabo a través del método cualitativo de Análisis del Discurso, que muestra los sentidos y significados que sobre universidad pública, expresan los estudiantes de últimos semestres de los programas de pregrado modalidad a Distancia de la Universidad de Cartagena (Colombia), en el periodo comprendido entre los años 2011 a 2016.

\section{Metodología}

T a investigación estuvo orientada desde el método cualitativo Análisis del Discurso (AD), el cual permite "descubrir los significados de las cosas, interpretar lo mejor posible las palabras, los escritos, los textos, los gestos, $y$ en general el comportamiento humano (...) conservando la singularidad del contexto que hace parte" (Dilthey, citado en: Martínez, 2006:3). Teun Van Dijk, plantea que al realizar un análisis discursivo es necesario tener en cuenta que "el discurso como acción social ocurre en un marco de comprensión, comunicación e interacción que a su vez son partes de estructuras y procesos socioculturales más amplios" (citado por Silva, 2002:4). En ese sentido, se asume que los discursos expresados por los estudiantes en el contexto universitario hacen eco de los discursos de un contexto sociocultural e histórico más amplio. Por ello, para el desarrollo de la investigación se diseñó la siguiente propuesta metodológica.

Tabla 1: Metodología de Investigación

\begin{tabular}{|l|l|}
\hline Enfoque & Hermenéutico \\
\hline Tipo & Descriptivo \\
\hline Perspectiva & Holística \\
\hline Estrategia & Análisis del discurso \\
\hline Técnica de recolección de información & Entrevista semi-estructurada (aplicada a 36 estudiantes) \\
\hline Instrumentos & $\begin{array}{l}\text { Guía de entrevista, matrices para sistematización y software } \\
\text { Atlas Ti }\end{array}$ \\
\hline
\end{tabular}

Fuente: Investigación: Representaciones sociales de universidad pública y su incidencia en la desercion estudiantil. Caso Centro Tutorial San Juan Nepomuceno-Universidad de Cartagena. Autora: Zoahamy Ardila Ramos 2016. 
7 Gentilicio utilizado para denotar la pertenencia/procedencia de los estudiantes y egresados de la Universidad de Cartagena (Colombia).
El proceso metodológico seguido se realizó a través de tres fases: la primera correspondió a la recolección de información secundaria y primaria. Para la información secundaria se recopilaron bibliografías, documentos y artículos que hacían referencia a la categoría de representaciones sociales de universidad pública, procediendo luego a la elaboración de fichas bibliográficas y matrices temáticas, que fueron derivándose según subcategorías de esta. Cabe señalar que previamente se realizó una lectura de contexto que dio cuenta de hechos o situaciones que desde el punto de vista social, económico, cultural, ambiental y político se registraron en el municipio de San Juan Nepomuceno, lo cual contribuyó no solo a tener un marco contextual sino además a identificar los discursos ampliados de la sociedad local frente al tema de universidad pública, permitiendo contrastar los discursos de los entrevistados con estos.

La información primaria se recolectó mediante la aplicación de entrevistas semiestructuradas dirigidas a los estudiantes de sexto a décimo semestre vinculados a los programas de pregrado modalidad abierta y distancia del Centro Tutorial San Juan Nepomuceno de la Universidad de Cartagena (Colombia), considerando una muestra representativa de 36 estudiantes, de sexo masculino o femenino (3 por cada uno de los semestres). Luego en la fase dos (2) se procedió a la organización y análisis de la información clasificada de acuerdo a las categorías y subcategorías de análisis propuestas en la investigación, utilizando como apoyo la herramienta para procesamiento de datos cualitativos Atlas-Ti. Posteriormente en la fase final se establecieron los hallazgos resultantes de la investigación y se planteó el diseño de una propuesta de adaptación de la política de retención y permanencia de la Universidad de Cartagena (Colombia), a las a necesidades y características del contexto de influencia del Centro Tutorial San Juan Nepomuceno.

\section{Representaciones sociales de universidad pública, desde la voz de los estudiantes udeceistas ${ }^{7}$}

\section{Primeros hallazgos}

Las representaciones sociales sobre universidad pública, de los estudiantes de los programas a distancia del Centro Tutorial San Juan Nepomuceno, se configuraron desde la postura teórica de Moscovici (1979) entendiéndolas como el "conocimiento de sentido común que tiene por objetivo comunicar, estar al día y sentirse dentro del ambiente social, y que se originan en el intercambio comunicacional de los grupos sociales", dicho en términos más llanos es una forma de pensamiento social que se materializa en estereotipos, 
creencias, concepciones y simbologías construidas por los individuos en sus interrelaciones cotidianas; desde allí, se infirieron posiciones sobre la significación de la universalidad del derecho a la educación, como marco de referencia para comprender los sentidos de sus acciones.

A continuación se presenta una síntesis de los primeros hallazgos, relacionados con la categoría de estudio:

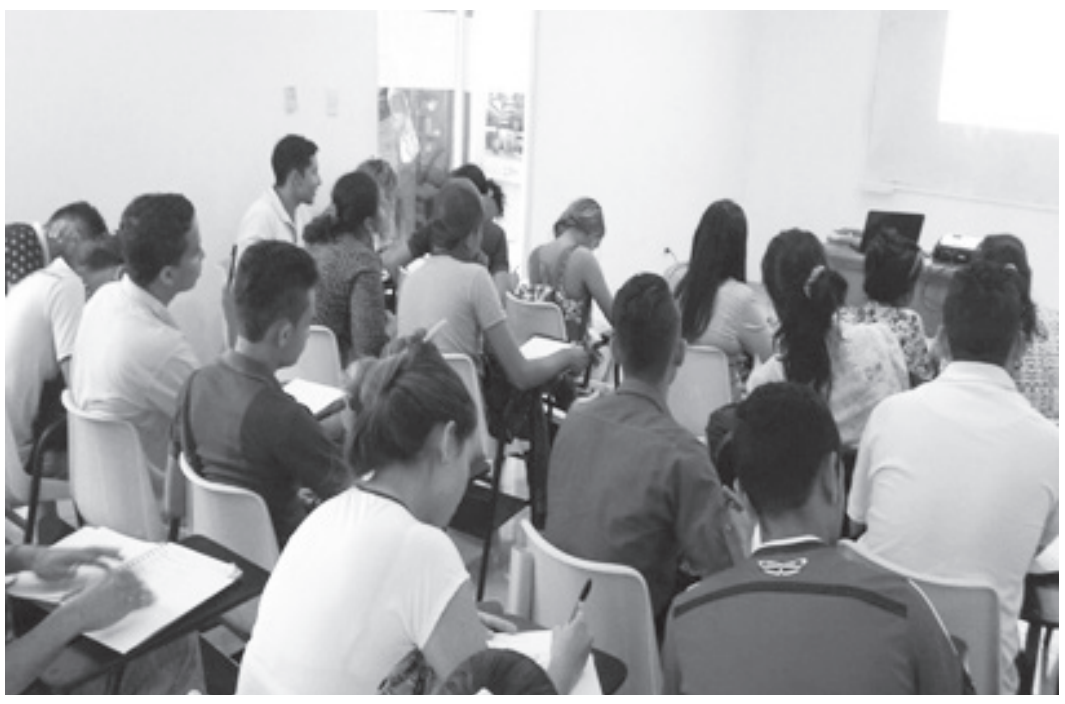

Centro Tutorial San Juan Nepomuceno - Bolívar. 2016 Foto: Zoahamy Ardila Ramos

Para los estudiantes, el estereotipo de universidad pública se ha construido solo desde el manejo administrativo y fuente de financiación y no desde el lugar del Estado como garante del derecho a la educación. Postura que se evidencia, en expresiones que dan cuenta del reconocimiento de la universidad pública como: "aquella que maneja el Estado" [... ], "cuenta con la financiación del Estado" [...], "aquella donde se paga poco", "aquella que se adapta a las personas de bajos recursos obteniendo un costo menor a lo que se pagaría en cualquier universidad privada" [...], "aquella que ofrece formación profesional a un costo que el ciudadano promedio pueda acceder". Como se puede apreciar, en estas expresiones, la representación social del rol de la universidad pública, conlleva a su desconocimiento como institución garante de derechos.

En contraposición a la postura anterior, algunos de los estudiantes han incorporado estereotipos que dan cuenta del reconocimiento de la universalidad del derecho a la educación, en tanto valoran en la universidad pública la función social de contribuir a la reducción de las desigualdades sociales y generación de equidad social; reconociéndola como: "la que brinda la oportunidad a todas las personas de estudiar, sin importar el tema económico ni social" , [...]"oportunidad y posibilidad de continuar estudios superiores a las personas que quieren salir adelante, formarse como profesionales, pero que no cuentan con los recursos económicos necesarios, postura que guarda estrecha relación con lo que plantea Baño (2010) "la Universidad que es de todos. Es Estatal porque el Estado es la expresión de la voluntad del todo nacional". De ahí, la importancia de la educación y en particular de la educación superior 
para el mejoramiento de la calidad de vida de los seres humanos y el desarrollo de los contextos sociales, en tanto, al elevar los niveles de competitividad de la población, se aumentan y potencializan los niveles de inserción al mercado laboral, así como las oportunidades de generación de ingresos y la capacidad de afrontar la satisfacción de necesidades básicas de los núcleo familiares en el Caribe colombiano.

Otro de los estereotipos de universidad pública se configuran desde el reconocimiento de la calidad como una característica connatural a la oferta pública educativa, en tanto el derecho no se limita solo al acceso, sino a la calidad del servicio: "la escogí por los altos niveles de calidad en educación que posee y por lo que es una de las mejores del país", [... "Escogí la Universidad de Cartagena ya que es una de las mejores del Caribe colombiano y su nivel de educación es muy bueno formando profesionales capaces de desempeñarse muy bien en su área, con los conocimientos adquiridos", [...] "es una universidad de prestigio y renombre, muy reconocida en el país" [...] "escogí la Universidad de Cartagena porque me brindó el beneficio de estar situado en este municipio", porque vivo aquí en San Juan y se nos hace más fácil estudiar y cumplir nuestras metas y propósitos" [...].

De esta forma, los estudiantes en la construcción de su representación social de universidad pública, han avanzado en la configuración del estereotipo de esta como garante del derecho a la educación, cuando reconocen cómo desde la oferta académica del Centro Tutorial San Juan Nepomuceno, la Universidad de Cartagena de acuerdo al compromiso asumido con la gente y la sociedad, aporta a los propósitos de aumentar la cobertura para cerrar las brechas entre zonas rurales y urbanas y contribuir a la construcción de ambientes de paz, donde todos gocen de los mismos derechos y oportunidades, en tanto favorece la participación de las minorías en la formación de capital humano.

Con relación a las concepciones de los estudiantes sobre la modalidad de educación a distancia como elemento configurador en la construcción de la representaciones sociales de universidad pública hacen alusión a esta como "una buena oportunidad para que las personas tengan acceso a la educación" [...], "una buena opción para quienes trabajan" [...] "una opción para las personas de escasos recursos que tienen que trabajar y estudiar simultáneamente", [...] "oportunidad de educarse desde el municipio sin necesidad de viajar a otras partes" [...] "es la oportunidad que se ofrece a las personas que viven en zonas de difícil acceso", de esto Claudine Herzlich (1979) anota que "la exigencias sobre el 
individuo que las circunstancias y las relaciones sociales imponen provocan una estimación, actuación o comunicación y llegan a ser fundamento de la conducta humana"; razón por la cual en los tres programas académicos del Centro Tutorial San Juan Nepomuceno, se aprecia una concepción que aporta a la construcción de una clara representación; favorable para garantizar la permanencia de los estudiantes en el sistema de educación superior hasta su titulación profesional.

En relación a la calidad educativa, los estudiantes, manejan la creencia de que son de calidad porque "ofrece una formación de calidad que hace de los estudiantes profesionales capaces de enfrentar el día a día en el campo profesional", [...] "los tutores están capacitados para enseñar y preparar excelentes profesionales en las diferentes áreas" [...], "son especializados en las materias", [...] "porque nosotros como estudiantes tenemos el empeño de aprender para que nuestra formación sea de calidad", [...] "me siento satisfecha con lo que he aprendido y todo lo que me ha brindado y enseñado esta universidad", "porque cuenta con una excelente infraestructura y los recursos necesarios para formar profesionales" [...] "porque maneja estándares de calidad que fortalecen sus programas".

Este reconocimiento de la trayectoria y prestigio educativo a la universidad pública, constituye para los estudiantes, garantía para asumir hasta culminar la formación profesional, los riesgos que la vida cotidiana le presenta de desertar. Por ello, y parafraseando a Abric (1971) a través de nuestro sistema de creencias y valores damos significado y coherencia a nuestro modelo del mundo, al que estamos profundamente vinculados. Cuestionar una de nuestras creencias puede desestabilizar todo el sistema al afectar a aquellas otras que se derivan o están relacionadas con ella. Esta es la razón por la que somos muy reacios, en muchas ocasiones, a modificar alguna de nuestras creencias. Cuando una creencia se instala en nosotros de forma sólida y consistente, nuestra mente elimina o no tiene en cuenta las experiencias que no casan con ella.

En cuanto a la función social que cumple la universidad en el municipio de San Juan, los estudiantes tienen la representación de "Una universidad que brinda la gran oportunidad de salir adelante", "una universidad que exige muchos sacrificios para obtener el éxito", [...] "una buena herramienta para formarse como persona y tener la mejor educación", "oportunidad de crecer personal y profesionalmente", [...] "una gran oportunidad de hacer realidad 
el sueño de ser profesional", preparación de los profesionales del futuro", [...] "Una herramienta para salir adelante en mi vida profesional", [...] "progreso, seriedad, respeto, oportunidad de cumplir mi sueño profesional", [...] "la oportunidad de ser un buen profesional a futuro", "simboliza la esperanza para salir adelante", Mi futuro, ser una excelente profesional para brindarle lo mejor de mí a la sociedad en especial a mi familia", [...] "ser parte de una familia que nos impulsa directo al triunfo". La calidad y el prestigio de la universidad pública, expresada en la figura de la Universidad de Cartagena, constituye el pilar fundamental para la formación del orgullo y dignidad Udeceista.

Es decir en la simbología de la condición Udeceista está expresado el sentido de pertenencia a una excelente universidad, a una universidad con altos estándares de calidad, a una universidad de gran prestigio en el Caribe colombiano, a una de las mejores universidades públicas del país, una universidad con acreditación de alta calidad.

Este gran reconocimiento, unido a la prospectiva laboral que genera en ellos el egresar de una institución de tan altas cualidades, se convierte en el motor que impulsa y motiva a los estudiantes para no desertar de sus programas, en tanto implica la pérdida de la condición de Udeceista que lleva con tanto orgullo, al tiempo que implica una renuncia a la oportunidad de mejoramiento a futuro de su calidad de vida personal y familiar.

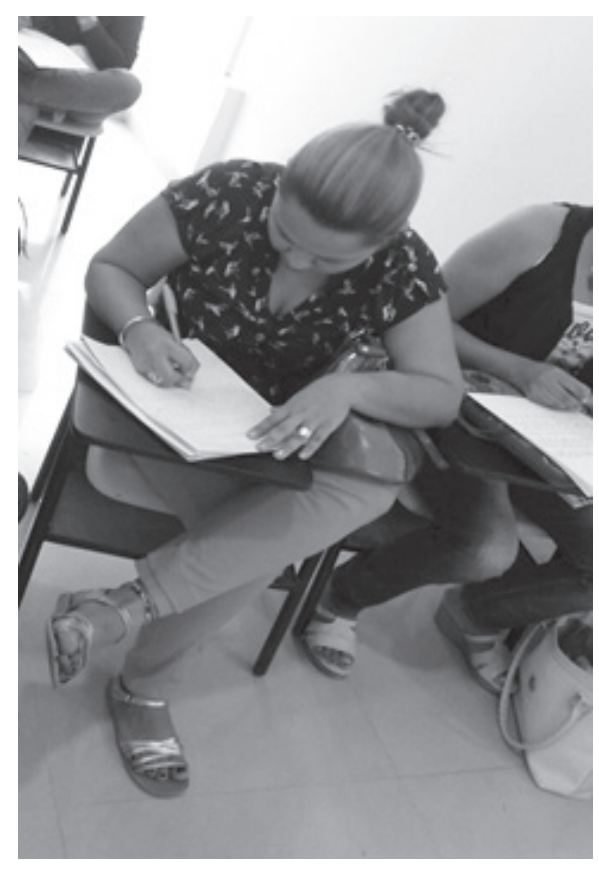

Todo lo anterior guarda estrecha relación con lo planteado por Maslow (1976) cuando plantea que los seres humanos no vivimos la realidad en sí, sino una elaboración mental de la misma. Lo que hace que la vida sea un constante manantial de esperanza y ricas alternativas o una inevitable fuente de sufrimiento. Lo que vivimos tal como lo vivimos, depende más de la representación y elaboración de nuestro mapa mental, que del territorio "real" en sí.

En el contexto la universidad pública es entendida como una institución, imprescindible para el desarrollo de la vida social de los sujetos en la búsqueda del bienestar común, en este caso la formación es el escenario para el saber y ejercicio de la ética civil, en la búsqueda de equidad y ciudadanía. Parafraseando al profesor Alfonso Conde (2011) la universidad pública es 
"Una entidad educativa": Ello significa que el objetivo central de su labor es el ser humano y su potencial de desarrollo como individuo y como partícipe de una colectividad. "De orden superior": Ello significa que más allá de instruir o adiestrar, tiene la misión de contribuir a la construcción de la capacidad individual y colectiva de transformación y creación.

En tal sentido, las representaciones sociales permiten a los estudiantes reconstruir la realidad y darle una significación específica, actuando como un marco de referencia que permite a estos comprender las situaciones y dar sentido a sus acciones, lo que se conjuga, según esta propuesta como una guía para la acción; lo que a su vez permite la apertura de debates y reflexiones en torno a las representaciones de universidad pública desde el enfoque de derechos, apostando a la contrucción de posturas críticas y reflexivas de los estudiantes universitarios de la Universidad de Cartagena y por qué no de la región Caribe.

Lo anterior solo será posible, desde el fortalecimiento de las estrategias y mecanismos que permitan consolidar la visión de la educación pública como un bien colectivo y un derecho social de todo ciudadano colombiano, que redunden en procesos de adaptación, retención y permanencia de los estudiantes en el sistema educativo.

\section{A manera de cierre}

raya Umaña, plantea que las representaciones sociales en definitiva:
"constituyen sistemas cognitivos en los que es posible reconocer la
presencia de opiniones, creencias, valores y normas (...) se constituyen, a su vez, como sistemas de códigos, valores, lógicas clasificatorias, principios interpretativos y orientadores de las prácticas, que definen la llamada conciencia colectiva", la cual permea la forma en que los sujetos actúan en el mundo.

Es decir, los sujetos reconocen la realidad que les rodea mediante explicaciones, análisis que le dan a los procesos de comunicación y del pensamiento social. De allí que se afirme que las representaciones sociales sintetizan dichas explicaciones y en consecuencia, hacen referencia a un tipo específico de conocimiento que juega un papel crucial sobre cómo los sujetos piensan y dan sentido a su vida cotidiana.

La intencionalidad y los retos que se plantea la academia en el marco sociohistórico en que estamos los colombianos y en particular la región Caribe, 
es en la definición del tipo de prioridades educativas y las políticas públicas que deberán facilitar las acciones que cimentarán las bases de una paz territorial. Dentro de ellas, la transformación del sistema sugiere recuperación y construcción de espacios de paz; incluida por lo que será también desde ella misma, la plataforma para reconstruir humanísticamente, la fragilidad del tejido social con equidad e inclusión, como factor de desarrollo humano y movilidad social.

El reto frente a lo anterior, es la toma de conciencia que debe hacer la institución frente a la responsabilidad social que tiene con el desarrollo de la región, desde el diseño de estrategias contextualizadas y pertinentes para las necesidades de cada unidad académica y para cada región, sin que ello implique perdidas sustanciales en el sentido de pertenencia por la Institución. No solo es suficiente encontrar los factores de riesgo asociados a la deserción, es vital además identificar aquellos factores protectores que promueven la permanencia en cada Centro Tutorial y a partir de ellos encadenar las estrategias. Sin desconocer lo que plantea el Ministerio de Educación Nacional de Colombia (2015) entorno a la necesidad de "dar una buena lectura de contexto y perspectiva diferencial a las acciones, no se puede hablar de la región como un todo, cada uno de los territorios cuenta con condiciones particulares, que requieren de acciones focalizadas y pertinentes".

En el marco de los procesos de autoevaluación institucionales este tipo de estudios permite la apertura de debates y reflexiones, en torno a las representaciones sociales de lo público y de la configuración de discursos y prácticas de ciudadanía en universitarios, tanto en la formación profesional como en ejercicio profesional.

\section{REFERENCIAS BIBLIOGRÁFICAS}

Alasino, Nidia (2011). Alcances del concepto de representaciones sociales para la investigación en el campo de la educación. Revista Iberoamericana de Educación. Volumen 56. Pág. 1-11.

Álvaro, José L. (2010). Diccionario Crítico de las Ciencias Sociales .Universidad Complutense de Madrid. Recuperado de: http://www.ucm.es/info/eurotheo/diccionario/R/representaciones sociales.htm

Baño, R. (2010). ¿Qué es una universidad pública?. Recuperado de:http://www.facso.uchile.cl/ noticias/67245/que-es-una-universidad-publica. 
Carballeda, Alfredo Juan Manuel (2007). Escuchar las prácticas: La supervisión como proceso de análisis de la intervención en lo social. Buenos Aires: Editorial Espacio, pág. 88.

Colombia. Ley General de Educación (Ley 115 de 1994). Ministerio de Educación Nacional. Artículo 70.

Colombia. Corte Constitucional. Relatoría Sentencia T-068/12. Derecho fundamental a la educación-Reiteración de jurisprudencia. Recuperado de: http://Www.corteconstitucional.gov.co/ RELATORIA/2012/T-068-12.htm.

Colombia. Constitución Política. Título I. De Los Principios Fundamentales. Artículo 1. ․, Artículo $2^{\circ}$., Artículo $5^{\circ}$.

Colombia. Ministerio de Educación Nacional. Ley 0115 de febrero 8 de 1994 -Por la cual se expide la Ley General de Educación. Recuperado de: http://www.mineducacion.gov.co/1621/ article-85906.html

Colombia. Ministerio de Educación Nacional. Ley 30 de 1994. Título Primero. Capítulo I. Artículo1º

Colombia. Ministerio de Educación Nacional. Instituciones de Educación Nacional. Recuperado de: http://www.mineducacion.gov.co/1759/w3-article-231240.html. Febrero 5 de 2016.

Consejo Nacional de Rectores-Sistema Universitario Estatal Colombiano. El desfinanciamiento de las universidades estatales colombianas. Recuperado de: http://www.mineducacion. gov.co/1621/articles-341914_archivo_pdf.pdf. Diciembre de 2012.

Periódico Alma Mater. Universidad de Antioquia, Medellín Colombia. Mayo de 2011, P. 14.

Piñeres de la Ossa, Dora. La cátedra historia de Cartagena, mirar su historia para construir su futuro. Volumen 1. Cartagena de Indias: Editorial Antillas/ Editorial Universitaria.

Salinas, Marta et al. Las representaciones sociales sobre la evaluación de los aprendizajes. Revista Educación y Pedagogía. Número 46, Universidad de Antioquia, pág. 207. Recuperado de: http:// aprendeenlinea.udea.edu.co/revistas/index.php/revistaeyp/article/view/6 945/6371.

Seidmann, Susana; Thomé, Sandra; Di Iorio, Jorgelina \& Azzollini, Susana (2008). Representaciones sociales del trabajo docente. Una investigación... Documento de Trabajo N ${ }^{\circ} 209$, Universidad de Belgrano. Recuperado de: http://www.ub.edu.ar/investigaciones/dt_nuevos/209_ seidmann.pdf.

Tamayo V., Alfonso (2015). Sentido y significado de la universidad pública. Observatorio de la Universidad Colombiana. En http://www.universidad.edu.co/index.php/ensayos-acadcosmainmenu-81/12777-sentido-y-significado-de-la-universidad-pca. 